\title{
FLOW OF A MAXWELL LIQUID FLUID BETWE EN TWO ROTATING COAXIAL CONES HAVING THE SAME VERTEX
}

\author{
By D. K. Mohan Rao \\ (Department of Applied Mathematics, Indian Institute of Science, Bangalor $\epsilon-12,1$ idia) \\ Received September 24, 1962 \\ (Communicated by Dr. P. L. Bhatnagar, P.A.Sc.)
}

\section{INTRODUCTION}

THE motion of a Newtonian fluid and two classes of non-Newtonian fluids, namely, the Reiner-Rivlin fluid and the Rivlin-Ericksen fluid between two rotating coaxial cones having the same vertex has been investigated by Bhatnagar and Rathna ${ }^{1}$ taking into consideration the inertial terms in Oseen approximation. The main characteristics of the motions are the introduction of secondary flows. In the case of non-Newtonian fluids the secondary flow is divided into two distinct domains. The stream lines in the inner region are closed loops. In the outer region the fluid moves more or less parallel to the generator of the cone to which it is closest, moving towards the vertex near one cone and away from the vertex near the other cone. In the present note we shall consider a Maxwell fluid. ${ }^{2,3}$ We find that when the relaxation times are small the secondary flow characteristics are similar in nature as predicted by Bhatnagar and Rathna.

\section{The Viscosity Distribution}

The equation of state for the Maxwell fluid under consideration is given by

$$
\begin{aligned}
& p^{i k}=2 \int_{-\infty}^{t} \psi\left(t-t^{\prime}\right) \frac{\partial x^{i}}{\partial x^{\prime} m} \frac{\partial x^{k}}{\partial x^{\prime r}} e^{(1) m r}\left(x^{\prime}, t^{\prime}\right) d t^{\prime} \\
& \psi\left(t-t^{\prime}\right)=\int_{0}^{\infty} \frac{\mathrm{N}(\tau)}{\tau} e^{-(t-t) / \tau} d \tau
\end{aligned}
$$

where

$$
p^{\prime i k} \text { is the deviatoric stress tensor, }
$$

$e^{(1) m r}$ the rate of strain tensor 
$x^{\prime j}=x^{i j}\left(x^{i}, t, t^{\prime}\right)$ is the position at time $t^{i}$ of the material point of the fluid which is instantaneously at the point $x^{i}$ at time $t, \mathrm{~N}(\tau) d \tau$ the total viscosity of all the Maxwell elements with relaxation times between $\tau$ and $\tau+d \tau$.

The sum of the viscosities of all the Maxwell elements is $\eta_{0}$ so that

$$
\int_{0}^{\mathrm{T}} \mathrm{N}(\tau) d \tau=\eta_{0}
$$

$\mathrm{T}$ being the largest relaxation time.

We consider a relaxation spectrum in which viscosity $S \eta_{0}$ is spread on the portion of the $\tau$-axis between $\tau=\mathrm{O}$ and $\tau=\mathrm{T}$, excluding the origin.

$$
\begin{aligned}
& \mathrm{N}(\tau)=\eta_{0}(1-\mathrm{S}) \delta(\tau)+\mathrm{SN}_{\mathrm{v}}(\tau), \quad 0<\mathrm{S} \leq 1 \\
& \int_{0}^{\mathrm{T}} \tau \mathrm{N}(\tau) d \tau=a \mathrm{ST} \eta_{0}, \quad 0<\mathcal{L} \leq 1 .
\end{aligned}
$$

The distribution given above represents the three constant spectrum studied by Walters ${ }^{3}$ when $\alpha=1$.

\section{The Primary Motion ${ }^{1}$}

We assume the primary motion to be that of a Newtonian liquid with the neglect of inertial terms. Here the stream lines are circles with centres on the common axis.

The variables used are rendered dimensionless by means of a standard length $L$ and a standard velocity $\mathrm{L} \Omega$, with $\Omega=\left|\Omega_{1}\right|+\left|\Omega_{2}\right|, \Omega_{1}$ and $\Omega_{2}$ being the angular velocities of the inner and outer cones respectively.

In spherical polar co-ordinates with the origin at the common vertex, the radial and transverse velocities $u_{0}, v_{0}$ in a meridian plane and $w_{0}$ the velocity normal to the meridian plane are given by

$$
\begin{aligned}
& u_{0}=v_{0}=0 \\
& u_{0}^{\prime}=r \sin \theta\left[\mathrm{A}_{1}\left\{\log \tan \frac{\theta}{2}-\cot \theta \operatorname{cosec} \theta\right\}+\mathrm{A}_{2}\right] \\
& \mathrm{A}_{1}=\frac{\Omega_{1}-\Omega_{2}}{\Omega \mathrm{K}}
\end{aligned}
$$




$$
\begin{aligned}
& \mathrm{A}_{2}=\frac{1}{\Omega \mathrm{K}} {\left[\Omega_{1}\left(\cot \theta_{2} \operatorname{cosec} \theta_{2}-\log \tan \frac{\theta_{2}}{2}\right)\right.} \\
&\left.+\Omega_{2}\left(\log \tan \frac{\theta}{2}-\cot \theta_{1} \operatorname{cosec} \theta_{1}\right)\right] \\
& \mathbf{K}=\log \left(\frac{\tan \frac{\theta_{1}}{2}}{\tan \frac{\theta_{2}}{2}}\right)+\cot \theta_{2} \operatorname{cosec} \theta_{2}-\cot \theta_{1} \operatorname{cosec} \theta_{1}
\end{aligned}
$$

$\theta_{1}$ and $\theta_{2}$ being the semi-vertical angles of the inner and outer cones respectively.

\section{The Secondary Motions}

We shall now study the modification of this primary flow due to the inclusion of the inertial terms and visco-elasticity regarding them as secondary effects.

Following Oseen's scheme, to take inertial terms into consideration in a limited manner, we have the following equations for the determination of the secondary motions.

The equation of continuity

$$
\frac{\partial u}{\partial r}+\frac{2 u}{r}+\frac{1}{r} \frac{\partial v}{\partial \theta}+\frac{v \cot \theta}{r}=0
$$

The equations of momentum

$$
\begin{aligned}
& -\frac{\mathrm{R}}{r}\left(w_{0}^{2}+2 w_{0} w\right) \\
& =-\frac{\partial p}{\partial r}+\Delta u-\frac{2 u}{r^{2}}-\frac{2}{r^{2}} \frac{\partial v}{\partial \theta}-\frac{2 v}{r^{2}} \cot \theta \\
& \quad-\frac{2 a \mathrm{ST}}{r} \sin ^{2} \theta\left(\frac{d \omega_{0}}{d \theta}\right)^{2} \\
& -\frac{\mathrm{R} \cot \theta}{r}\left(w_{0}^{2}+2 w_{0} w\right) \\
& =-\frac{\partial p}{\partial \partial \theta}+\Delta v+\frac{2}{r^{2}} \frac{\partial u}{\partial \theta}-\frac{v}{r^{2}} \operatorname{cosec}^{2} \theta
\end{aligned}
$$




$$
\begin{gathered}
-\frac{2 a \mathrm{ST}}{r} \cos \theta \sin \theta\left(\frac{d \omega_{0}}{d \theta}\right)^{2} \\
\frac{\mathbf{R}}{r}\left(v \frac{d w_{0}}{d \theta}+2 u w_{0}+v w_{0} \cot \theta\right)=\Delta w-\frac{w}{r^{2} \sin ^{2} \theta}
\end{gathered}
$$

where

$$
\Delta \equiv \frac{\partial^{2}}{\partial r^{2}}+\frac{2}{r} \frac{\partial}{\partial r}+\frac{\cot \theta}{r^{2}} \frac{\partial}{\partial \theta}+\frac{1}{r^{2}} \frac{\partial^{2}}{\partial \theta^{2}},
$$

$p$ is the hydrostatic pressure corresponding to the secondary flows. $u, v$ and $w=r \sin \theta \omega$ are the velocities in the secondary motion in directions parallel to $u_{0}, v_{0}, w_{3}, \overline{\mathrm{T}}=\Omega \mathrm{T} \mathrm{R}=\mathrm{L}^{2} \Omega / \nu, \nu$ being the kinematic coefficient of viscosity, and we have taken $\overline{\mathrm{T}}$ to be of the order of the square of the Reynolds Number $\mathbf{R}$ and neglected terms like $\mathbf{R}^{3} u, \mathbf{R}^{3} v$ in comparison with terms like $\mathrm{R} u, \mathbf{R} v$.

Equations (4.1)-(4.4) are to be solved with the boundary conditions

$$
u=v=w=0 \quad \text { when }\left\langle\begin{array}{l}
\theta=\theta_{1} \\
\theta=\theta_{2}
\end{array}\right.
$$

Following Bhatnagar and Rathna, we assume solutions of the form

$$
\begin{aligned}
& u=-\frac{1}{r^{2} \sin \theta}\left[\mathrm{R} \frac{\partial \psi_{1}}{\partial \theta}+\mathrm{K} \frac{\partial \psi_{2}}{\partial \theta}\right] \\
& v=\frac{1}{r \sin \theta}\left[\mathrm{R} \frac{\partial \psi_{1}}{\partial r}+\mathrm{K} \frac{\partial \psi_{2}}{\partial r}\right] \\
& \omega=\mathrm{R}^{2} r^{4} \sin \theta \nsim \\
& p=\mathrm{R} p_{1}+\mathrm{K} p_{2}
\end{aligned}
$$

where the suffixes 1 and 2 correspond to the secondary flows in a meridian plane induced by the inclusion of the inertial terms and visco-elasticity respectively, and $\mathrm{K}=2 a \mathrm{ST}$.

Writing

$$
\begin{aligned}
& \psi_{1}=r^{5} \mathrm{~F}_{1}(\theta) \\
& \psi_{2}=r^{3} \mathrm{~F}_{2}(\theta) \\
& p_{1}=r^{8} \tilde{\phi}_{1}(\theta)
\end{aligned}
$$


and

$$
p_{2}=c \log r+\tilde{p}_{2}(\theta)
$$

$C$ being a constant,

we have the following equations for the determination of $F_{1}(\theta)$ and $F_{2}(\theta)$ :

$$
\begin{aligned}
\mathrm{D}^{4} \mathrm{~F}_{1}(\theta) & -2 \cot \theta \mathrm{D}^{3} \mathrm{~F}_{1}(\theta)+\left(28+3 \cot ^{2} \theta\right) \mathrm{D}^{2} \mathrm{~F}_{1}(\theta) \\
& -\cot \theta\left(29+3 \cot ^{2} \theta\right) \mathrm{DF}_{1}(\theta)+120 \mathrm{~F}_{1}(\theta) \\
=4 \mathrm{~A}_{1} & {\left[\mathrm{~A}_{1}\left(\log \tan \frac{\theta}{2}-\cot \theta \operatorname{cosec} \theta\right)+\mathrm{A}_{2}\right] }
\end{aligned}
$$

and

$$
\begin{aligned}
& D^{4} F_{2}(\theta)-2 \cot \theta D^{3} F_{2}(\theta)+\left(8+3 \cot ^{2} \theta\right) D^{2} F_{2}(\theta) \\
& \quad-\left(9+3 \cot ^{2} \theta\right) \cot \theta \mathrm{DF}_{2}(\theta)=16 \mathrm{KA}_{1}^{2} \cot \theta \operatorname{cosec}^{3} \theta(4 \cdot 9)
\end{aligned}
$$

where

$$
\mathrm{D} \equiv \frac{d}{d \theta} .
$$

We can easily check that

$$
\begin{aligned}
\mathrm{F}_{1}(\theta)= & a_{1}\left[7 \cos ^{5} \theta-10 \cos ^{3} \theta+3 \cos \theta\right] \\
+ & a_{2}\left[\left(7 \cos ^{5} \theta-10 \cos ^{3} \theta+3 \cos \theta\right) \log \tan \frac{\theta}{2}+7 \cos ^{4} \theta\right. \\
& \left.-\frac{23}{3} \cos ^{2} \theta+\frac{16}{15}\right] \\
+ & a_{3}\left[\cos ^{3} \theta-\cos \theta\right]+a_{4}\left[\left(\cos ^{3} \theta-\cos \theta\right) \log \tan \frac{\theta}{2}\right. \\
+ & \left.\cos ^{2} \theta-\frac{2}{3}\right] \\
+ & \mathrm{A}_{1}{ }^{2}\left[\log ^{2} \tan \frac{\theta}{2}\left(-\frac{9}{8} \cos ^{5} \theta+\frac{7}{4} \cos ^{3} \theta-\frac{5}{8} \cos \theta\right)\right. \\
+ & \log \tan \frac{\theta}{2}\left(-\frac{9}{4} \cos ^{4} \theta+\frac{11}{4} \cos ^{2} \theta-\frac{1}{2}\right) \\
& \left.-\frac{63}{80} \cos ^{5} \theta+\frac{2}{7} \cos ^{3} \theta+\frac{211}{560} \cos \theta\right]+\frac{\mathrm{A}_{1} \mathrm{~A}_{2}}{30}
\end{aligned}
$$


Flow of Maxwell Liquid Fluid between Two Rotating Coaxial Cones 203 and

$$
\begin{gathered}
\mathrm{F}_{2}(\theta)=b_{1} \cos \theta+b_{2}+b_{3}\left(\cos ^{3} \theta-\cos \theta\right) \\
+b_{4}\left[\cos ^{2} \theta+\left(\cos ^{3} \theta-\cos \theta\right) \log \tan \frac{\theta}{2}\right] \\
+\mathrm{K} \\
{\left[\left(\cos \theta+\log \tan \frac{\theta}{2}\right)\left(1-2 \cos ^{2} \theta\right)\right.} \\
\left.+\left(\cos \theta-\cos ^{3} \theta\right) \log ^{2} \tan \frac{\theta}{2}\right]
\end{gathered}
$$

where the constants $a$ 's and $b$ 's have to be determined from the boundary conditions (4.5).

\section{The Stresses}

The pressure $p$ is given by (4.6) and (4.7) with

$$
\begin{aligned}
& 2 \bar{p}_{1}(\theta)=\sin ^{2} \theta \omega_{0}^{2}-\operatorname{cosec} \theta\left[\left(21+\cot ^{2} \theta\right) \mathrm{DF}_{1}(\theta)-\cot \theta \mathrm{D}^{2} \mathrm{~F}_{1}(\theta)\right. \\
& \left.\quad+\mathrm{D}^{3} \mathrm{~F}_{1}(\theta)\right] \\
& c=3 b_{1}
\end{aligned}
$$

and

$$
\tilde{p}_{2}(\theta)=4 \mathrm{~A}_{1}{ }^{2} \operatorname{cosec}^{4} \theta+\operatorname{cosec} \theta \mathrm{DF}_{2}(\theta)+6 \int \mathrm{F}_{2}(\theta) \operatorname{cosec} \theta d \theta .
$$

The stress components are given by

$$
\begin{aligned}
p_{r r}=-p & -6 \mathrm{R} r^{2} \operatorname{cosec} \theta \mathrm{DF}_{1}(\theta)-2 \mathrm{~K} \operatorname{cosec} \theta \mathrm{DF}_{2}(\theta) \\
p_{\theta \theta}=-p & +2 \mathrm{R} r^{2} \operatorname{cosec} \theta\left[4 \mathrm{DF}_{1}(\theta)-5 \mathrm{~F}_{1}(\theta)\right] \\
& \quad+2 \mathrm{~K} \operatorname{cosec} \theta\left[2 \mathrm{DF}_{2}(\theta)-\mathrm{F}_{2}(\theta) \cot \theta\right] \\
p_{\phi \phi}=-p & +2 \mathrm{R} r^{2} \operatorname{cosec} \theta\left[5 \mathrm{~F}_{1}(\theta) \cot \theta-\mathrm{DF}_{1}(\theta)\right] \\
& \quad+2 \mathrm{~K} \operatorname{cosec} \theta\left[2 \mathrm{~A}_{1}^{2} \operatorname{cosec}^{3} \theta+3 \cot \theta \mathrm{F}_{2}(\theta)-\mathrm{DF}_{2}(\theta)\right] \\
p_{r \phi}= & \mathrm{R} r^{2} \operatorname{cosec} \theta\left[10 \mathrm{~F}_{1}(\theta)+\cot \theta \mathrm{DF}_{1}(\theta)-\mathrm{D}^{2} \mathrm{~F}_{1}(\theta)\right] \\
& \quad+\mathrm{K} \operatorname{cosec} \theta\left[\cot \theta \mathrm{DF}_{2}(\theta)-\mathrm{D}^{2} \mathrm{~F}_{2}(\theta)\right] \\
p_{\theta \phi}= & \mathrm{R}^{2} r^{4} \sin \theta \mathrm{D} \approx(\theta) \\
p_{r \phi}= & 4 \mathrm{R}^{2} r^{4} \sin \theta \approx(\theta) .
\end{aligned}
$$




\section{NUMERICAL WORK}

We have obtained the values of the constants $a$ 's and $b$ 's in the case when

$$
\Omega_{1}=1, \Omega_{2}=0, \theta_{1}=\frac{\pi}{3}, \theta_{2}=\frac{\pi}{2}, \alpha=1, \quad \mathrm{~S}=0.5
$$

and

$$
\overline{\mathbf{T}}=0.01 \text {. }
$$

The method is quite general and other cases may be similarly discussed. The values of $\alpha, \mathrm{S}$ and $\overline{\mathrm{T}}$ correspond to an idealized visco-elastic fluid studied by Walters. ${ }^{3}$

The values of the constants $a$ 's and $b$ 's are given by

$$
\begin{aligned}
& a_{1}=-0.3002735 \mathrm{~A}_{1}^{2} \\
& a_{2}=0.003091365 \mathrm{~A}_{1}^{2} \\
& a_{3}=-0.02403479 \mathrm{~A}_{1}^{2} \\
& a_{4}=0.00494618 \mathrm{~A}_{1}^{2} \\
& b_{1}=b_{3}=-1.5866184 \mathrm{~A}_{1}{ }^{2} \\
& b_{2}=0 \text { and } \\
& b_{4}=0.0467785 \mathrm{~A}_{1}{ }^{2} .
\end{aligned}
$$

The stream lines for the secondary flow are given by

$$
\psi=\mathbf{R} \psi_{1}+\mathbf{K} \psi_{2}=\text { constant. }
$$

Figure 1 shows the stream lines in the present case for

$$
\psi= \pm 10^{-8} \times 7 \cdot 5, \pm 10^{-8} \times 8 \text {. }
$$

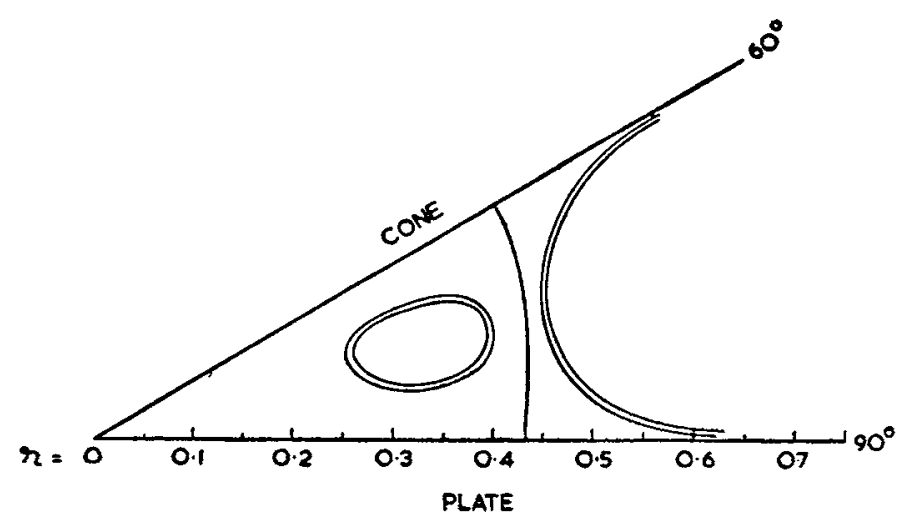

Frg. 1. Stream Lines of the Secondary Flow when $R=0.1$ and $\bar{T}=0.01$. 


\section{DisCussion OF THE RESUlts}

We observe that the flow field is divided into two domains by a stream line. The dividing stream line starts normally to the cone and the plate and the radius vector drawn from the vertex to the dividing stream line goes on decreasing as we move from the cone to the plate. It appears that the circular dividing stream line as reported by Bhatnagar and Rathna ${ }^{1}$ is the effect of the assumption of small angle between the cones.

In the region near the vertex the stream lines are closed loops. In the outer region the fluid is drawn towards the vertex near the stationary plate and is thrown away from the vertex near the rotating cone. In general, then, the secondary flow resembles that reported by Bhatnagar and Rathna ${ }^{1}$ if we make allowance for the smaller angle between the cones.

\section{SUMMARY}

We consider the secondary flows arising in the motion of a Maxwell fluid between two rotating coaxial cones having the same vertex. We find that in any meridian plane passing through the common axis of the cones, the flow field is divided into two regions. Such a division of flow field was first reported by Bhatnagar and Rathna. ${ }^{1}$

\section{ACKNOWLEDGEMENTS}

The author wishes to express his sincere gratitude to Prof. P. L. Bhatnagar for suggesting this investigation and for his kind help and guidance during the preparation of this paper.

\section{REFERENCES}

1. Bhatnagar, P. L. and Rathna, Quart. Journ. Mech. and Appl. Maths. (To be published). S. L.

2. Oldroyd, J. G.

.. Proc. Roy. Soc., 1950, 200 A, 523.

3. Walters, $\mathbf{K}$.

.. Quart. Journ. Mech. and Appl. Maths., 1962, 15, 76. 